\title{
Relationship between global mean sea-level and global mean temperature in a climate simulation of the past millennium
}

\author{
Hans von Storch • Eduardo Zorita • \\ Jesús F. González-Rouco
}

Received: 30 April 2008 / Accepted: 27 July 2008 / Published online: 22 August 2008

(C) The Author(s) 2008. This article is published with open access at Springerlink.com

\begin{abstract}
The possibility of using global mean nearsurface temperature, its rate of change or the global mean ocean heat-flux as predictors to statistically estimate the change of global mean sea-level is explored in the context of a long climate simulation of the past millennium with the climate model ECHO-G. Such relationships have recently been proposed to by-pass the difficulty of estimating future sea-level changes based on simulations with coarse-resolution climate models. It is found that, in this simulation, a simple linear relationship between mean temperature and the rate of change of sea level does not exist. A regression parameter linking both variables, and estimated in sliding 120-year windows, varies widely along the simulation and, in some periods, even attains negative values. The ocean heat-flux and the rate-of-change of mean temperature seem to better capture the rate-ofchange of sea level due to thermal expansion.
\end{abstract}

Keywords Global sea-level • Temperature • Last millennium - Climate simulation • Climate change

Responsible Editor: Joerg-Olaf Wolff

H. von Storch · E. Zorita $(\varangle)$

GKSS Research Centre, Geesthacht, Germany

e-mail: eduardo.zorita@gkss.de

H. von Storch · E. Zorita

KlimaKampus, Hamburg, Germany

H. von Storch

e-mail: hvonstorch@web.de

J. F. González-Rouco

Depto. de Astrofśica y CC. Atmósfera, U. Complutense,

Madrid, Spain

\section{Introduction}

The physical mechanisms that cause global sea-level variations at decadal timescales mainly comprise the changes in water density due to changes in water temperature and salinity, and the increase of ocean water mass due to melting of land glaciers and polar ice caps. Estimations of future global sea-level rise brought about by increasing concentrations of atmospheric greenhouse gases of anthropogenic origin are based on simulations with coarse-resolution global climate models, which include a representation of the most important components of the climate system, the atmosphere, the ocean and the cryosphere. The coarse horizontal resolution of current climate models, roughly $200 \times 200 \mathrm{~km}$, imposes some limitations on the skill of future projections of global sea-level rise. Some of the processes that modulate the heat flux into the ocean, such as convection, may not be adequately represented in ocean models. Also, land-locked glaciers, with extensions of a few kilometres, often located in areas of complex topography, are only very crudely represented in the soil sub-models, which operate at a resolution that usually matches the resolution of the atmospheric models. A further complication stems from the ice dynamics in the polar ice sheets and its response to an increased heat flux in the future. This dynamics, far from being a simple heat-uptake from the atmosphere, may be modulated by the presence of ice cracks, melt water flow within the ice and ice sliding over the bedrock that supports the ice caps. Due to these reasons, the Fourth Assessment report of the Intergovernmental Panel on Climate Change (Meehl et al. 2007) included estimations of future projections of sea-level rise with wide uncertainty ranges, and it was 
very cautious about the question of the magnitude of the sea-level rise component due to the melting of the polar ice caps.

However, decision makers require information about the possible range of sea-level rise that include all possible components for long-term planning with a time horizon of 100 years or longer. To fulfil this need and partially by-pass the uncertainties associated to the representation of the above processes in climate models, ad hoc (sometimes labelled "semi-empirical") methods have been proposed to estimate future global mean sea level rise based on the global mean temperature change simulated by climate models. Recently, Rahmstorf (2007a), hereafter R07, proposed a linear relationship between the rate of global mean sealevel rise $\frac{d H}{d t}$ and the global mean near-surface airtemperature deviations (see also Katsman et al. 2008). This relationship is calibrated with observed data, thus incorporating in a somewhat realistic and condensed manner all known and unknown mechanisms modulating the global sea-level height. As it is generally assumed that climate models are much more skilful in simulating future changes of the global mean temperature, such an ad hoc relationship would perhaps allow for more reliable estimations of future sea-level rise and of their uncertainties.

As with any empirical-statistical relationship, it is assumed that the same physical mechanisms operating in the present climate will operate in the future, even when the predictors and the predictand will attain values outside the range observed in the calibration range. It is, of course, not possible to test the validity of this assumption in the future. One strategy is to test the statistical methods in the virtual reality produced in simulations with state-of-the-art climate models. This line of research has also been exploited before to test the skill of statistical methods of proxy-based reconstructions of past climates (see, e.g. Lee et al. 2008). Following this strategy, we test in this paper several hypotheses concerning the relationship between global mean sea level and other thermal surface variables in a long climate simulation of the past millennium with the climate model ECHO-G driven by estimations of past greenhouse gas, volcanic and solar forcing. The rationale of using this simulation as a zero-order test bed is to ascertain whether the statical relationships are stable in time in a climate that does not deviate very strongly from the present one and does not show rapid climate changes.

\section{Semi-empirical models linking global mean sea level and global mean surface thermal variables}

The method proposed in $\mathrm{R} 07$ relates the rate of change of sea-level height $\mathrm{H}$ with the global mean temperature deviations from a reference level $T-T 0$ :

$$
\frac{d H}{d t} \propto a(T-T 0)
$$

The coefficient $a$ is estimated from the observational record of the past 120 years (1880-2000) by ordinary least squares. Once estimated, the statistical model (Eq. 1) can be applied to projections of future global temperature change simulated by a climate model and, assuming that the linear relationship would still hold in the future, an estimation of global mean sea-level rise can be obtained.

The justification for the conceptual model (Eq. 1) is based on the argument according to which sea level in a stationary state, after forced by a sudden temperature change, will adjust exponentially to a new stationary state (see Fig. 1 in Rahmstorf 2007a). This adjustment would follow a decay-type function $g\left(t-t_{0}\right)$ with a typical timescale of the order of several hundred years, reflecting the time required by a heat pulse to reach the deeper ocean layers and the time needed by land-ice to melt. The initial rate of the sea-level adjustment $\frac{d g}{d t}$ would be proportional to the magnitude of the sudden temperature change, from which the linear relationship between $T$ and rate of sea-level rise follows.

The ad-hoc approach of R07 has been subject to some criticism on statistical grounds/space/(Schmith et al. 2007). One important caveat is the non-stationary, or near non-stationary, character of the timeseries of global mean near-surface temperature and global mean sea-level. The relatively short record length and the presence of stochastic trends, that impinge a longmemory timescale on the time series, may jeopardise the estimation of regression parameters $a$ based on ordinary least-squares methods. When the input data used to estimate a linear relationship exhibit temporal correlation [as described by auto-regressive processes with a high degree of autocorrelation or even by nonstationary (so called integrated) processes], other methods, such as co-integration analysis, have to be applied to ascertain whether a linear relationship exists and, in that case, to estimate the regression parameter (see, e.g. Kaufmann and Stern 2002 for the application of 
co-integration analysis in a climate research context). Schmith et al.'s (2007) analysis demonstrates that this happens in the case of sea level change data. In the case of non-stationary timeseries, the conventional estimation of the parameter $a$ leads to misleading results. This problem is compounded by the use of heavily smoothed data that, in a short observational record, include only a small number of degrees of freedom (Rahmstorf 2007b).

From the physical point of view, the statistical model (Eq. 1) may not appear completely straightforward. Global mean temperature does not change stepwise from one stationary state to another but follows a continuous and smooth evolution $T(t)$. Still in the framework of R07, rather than being caused by the instantaneous deviations of the global mean temperature, global sea-level at a certain time $t$ will be more accurately described by the mathematical convolution of the two functions: the temperature at all times previous to time $t, T(t-\Delta t)$ and the corresponding adjustment function $g(\Delta t)$. Also, ignoring for the moment the non-linear dependence of the thermal expansion of sea water with water temperature, the contribution of the thermal expansion to the rate of sea-level change $\frac{d H_{t e}}{d t}$ would not be directly linearly related to the water temperature - and, therefore, not to the near-surface air-temperature either - but to the global mean heatflux into the ocean $F$ :

$\frac{d H_{t e}}{d t} \propto F$

The proportionality constant has been denoted as the "expansion efficiency of heat" by Russell et al. (2000). Equation 2 should hold exactly, without any time lag, if the thermal expansion of seawater were independent of temperature. In general, it will depend on water temperature, and a lag will exist between the rate of change of sea level and temperature that will depend on the three-dimensional water temperature field and the rate of heat diffusion.

For model Eq. 1 to hold, at least approximately, a linear relationship between the heat flux $F$ and the near-surface air-temperature should exist, which does not seem directly obvious in view of the complexity of the processes that modulate the heat uptake by the ocean. However, previous analyses of future simulations with a suite of global climate models indicate that the heat-flux into the ocean is correlated with the global mean near-surface temperature (Gregory et al. 2001;
Katsman et al. 2008), at least in situations where the external forcing is steadily increasing. This indirectly supports the statistical model Eq. 1. However, the physical mechanisms that may give rise to this linear link remained to be explained in those analyses. Another possibility is that, in simulations with steadily increasing external forcing, both global mean temperature and global heat-flux appear correlated because of the longterm trends present in both variables. A separate analysis of the proportionality between $T$ and $F$ in some sub-periods in those simulations, e.g. in the twentieth and the twenty first centuries, would help to clarify this question.

Other plausible models for the thermal expansion component of sea level would be simply to assume a proportionality between deviations of the global mean sea-level and deviations of the global mean nearsurface temperature. This would stem from Eq. 2 by assuming that the latter is a proxy for the 3 -dimensional average of ocean temperature:

$H_{t e} \propto T$

From this statistical model, a relationship between the rates of change of global mean sea-level and of near-surface temperature can be also proposed:

$\frac{d H_{t e}}{d t} \propto \frac{d T}{d t}$

It is not easy to test which one of these statistical models may be more valid in the real world. Timeseries of estimations of global sea level based on satellite measurements start only in 1993 (Cazenave and Nerem 2004) and the estimations of global mean sea level based on tide gauges are burdened by the relatively sparse sampling in the first half of the twentieth century. The long memory properties of sea level variations also limit a proper inference when dealing with such data (Schmith et al. 2007).

An alternative strategy is to test these statistical models (Eqs. 1-4) in the virtual reality produced by simulations with global climate models. In these virtual, physically consistent realities, all data are known perfectly and the complete chain of reasoning leading to a semi-empirical estimation of sea-level rise - i.e. calibration of the statistical model in an pseudoobservational period, application to future climatechange scenario projections and comparison with the simulated sea level-can be emulated and the skill of the statistical model evaluated. This test is, of course, too 
optimistic since, in the real world, the temperature and sea-level data are affected by sampling and measuring uncertainties, which are absent in the model data; also, in the real world, more significant processes influence sea level and its spatial distribution.

Rahmstorf (2007a) tested the statistical model Eq. 1 with data from climate simulations with the model of intermediate complexity CLIMBER (Montoya et al. 2005 ) in the period 1880-2100 under the SRES emission scenario A1F1 (Meehl et al. 2007). The model CLIMBER does not represent the contribution of landice melting, so that only the statistical relationships between the thermal expansion component and the global mean temperature could be tested. The statistical model Eq. 1 delivered reasonable estimations of global mean sea level until about 2070, producing an overestimation of the global sea level from there onwards. In year 2100, the estimated global sea level rise was about $30 \%$ higher that the sea-level rise actually simulated by CLIMBER.

\section{Climate model and simulation}

This study analyses a simulation with the coupled atmosphere-ocean General Circulation Model ECHO$\mathrm{G}$, consisting of the atmospheric model ECHAM4 (horizontal resolution approx. $3.75^{\circ} \times 3.75^{\circ}$ ) and the oceanic component HOPE-G (Legutke and Voss 1999). The ocean model has a horizontal resolution of $2.8^{\circ} \times 2.8^{\circ}$ with an increasingly finer resolution in the tropical regions towards the equator, where it reaches a $0.5^{\circ} \times 0.5^{\circ}$ meridional separation. The ocean model has 19 levels in the vertical. A version of the ECHO$\mathrm{G}$ model, slightly modified to include the effect of anthropogenic aerosols in the twentieth century, has been included in the simulations for the Fourth Assessment Report of the IPCC (Meehl et al. 2007). The simulation analysed here covers the period 1000-1990 and was driven by the estimations of past external forcing until 1990 (solar variations, greenhouse trace-gas concentrations and volcanic activity). No anthropogenic aerosols or ozone changes were considered (González-Rouco et al. 2006). The external forcings were derived from the values provided by Crowley (2000). The variations in solar irradiance, implemented in the simulation as a single annual value independent of spectral band, were estimated from concentrations of ${ }^{10} \mathrm{Be}$ in ice cores and re-scaled to units of watts per square meter, so that the difference in solar irradiance between the mean of 1960-1990 and that of 1680-1710 (the Late Maunder Minimum) was $0.3 \%$. The volcanic forcing was also taken from the estimations provided by Crowley (2000) and translated to effective changes in the solar constant independent of latitude and with the same seasonal profile for all eruptions (peaking in northern summer). The concentrations of $\mathrm{CO}_{2}$ and $\mathrm{CH}_{4}$, to drive the model, were taken from ice-core-based estimations (Etheridge et al. 1996, 1998). The simulation used here is a second realisation of a simulation discussed in previous papers (e.g. von Storch et al. 2006). This former simulation may present some initial drift in the first 2-3 centuries due to initial conditions extracted from a present-day control run that could have been possibly too warm and not in equilibrium with the external forcing in year 1000 (Osborn et al. 2006). The present simulation was started from the initial conditions extracted from year $1700 \mathrm{AD}$. in the previous simulation (von Storch et al. 2006, 2008).

\section{Model sea-level data}

As for the CLIMBER model, the model ECHO-G does not represent changes in the volume of land-ice or polar ice caps. Therefore, long-term changes in this model's global mean sea-level are exclusively due to changes in the density of the water column, ignoring possible storage of water in the atmosphere or over land. The density of the water column was computed from the simulated in-situ modelled temperature and salinity according to the standard UNESCO formula for sea water (Fofonoff and Millard 1883). The resulting 2-dimensional sea-level field was globally averaged, taking into account the diminishing size with latitude of the model grid cells.

The statistical model used by (Rahmstorf 2007a) (model Eq. 1) uses estimated "non-linear trends" of the global mean sea level and global mean temperature. These trends were calculated in R07, based on the analysis by Jevrejeva et al. (2006), by the application of singular spectrum analysis (Allen and Smith 1996), a statistical technique related to principal components analysis, with an embedding dimension of 15 years. Basically, the output of singular spectrum analysis is a smoothed version of the original timeseries, whereby the filter characteristics are derived from the input data. The same method, with the same embedding dimension, was applied here to the ECHO-G data. The term non-linear trends might be confusing in some other context, perhaps interpreted as the estimation of a deterministic trend present in the data. This is not the case here, and in the following, we will also use the term "low-frequency variability" to underline the meaning of trend in this context. Although probably a simpler 
method to estimate the low-frequency behaviour of these timeseries would have yielded similar results, the method based on SSA has been applied here to facilitate the comparison with previous results, as it was the choice of 15-year-timescale degree of filtering.

This latter choice could be a compromise between the need to filter out the high-frequency variations of the temperature record, which are not followed by the more sluggish ocean response. The statistical analysis of the interannual co-variability would yield only very weak statistical links. On the other hand, a strong filtering considerably reduces the number of degrees of freedom in the data, thus making the estimations of the statistical parameters more uncertain. Again, the aim of the present analysis is not to find the optimal degree of filtering, although it is acknowledged that this question may be related to the choice of optimal predictor.

\section{Results}

The low-pass filtered time series of the model global mean thermosteric sea level and global mean temperature are displayed in Fig. 1a. In the following, model sea level will refer only to the thermosteric component, as explained in the previous section.

Both series show maximum values at the beginning and at the end of the simulation, which straddle three centuries - roughly between 1500 and 1800 - when both global means of temperature and sea level were lower than the long-term average. The simulation of the global mean temperature agrees well with other simulations of the same period with the climate system model CSM (Zorita et al. 2007). Unfortunately, sea-level data from these simulations have not been analysed and published yet. However, the simulated evolution of global ocean heat content is quite similar to the one obtained by Crowley et al. (2003) in a simulation with an energy-balance model (Fig. 2). The global mean sea level simulated by ECHO-G shows an increase in the last 200 years of the simulation of about $70 \mathrm{~mm}$ and a linear trend in the period 1955-1995 of $0.42 \pm$ $0.06 \mathrm{~mm} /$ year, which agrees quite well with estimation of the observed thermosteric contribution to global sea-level rise in the same period $(0.40 \pm 0.09 \mathrm{~mm} / \mathrm{year}$, Antonov et al. 2005).
Fig. 1 Time series derived from the simulation with the model ECHO-G in the past millennium related to global mean near-surface temperature $T$ and global mean thermosteric sea-level $H_{t e}$, total heat-flux into the ocean $F$ and the time derivatives of $T_{t e}$ and $H$ (a-d). The predictands $H$ and $\frac{d H}{d t}$ are shown with their physical units as simulated by the model ECHO-G; the other predictor variables are rescaled to have the same variance as the corresponding predictand
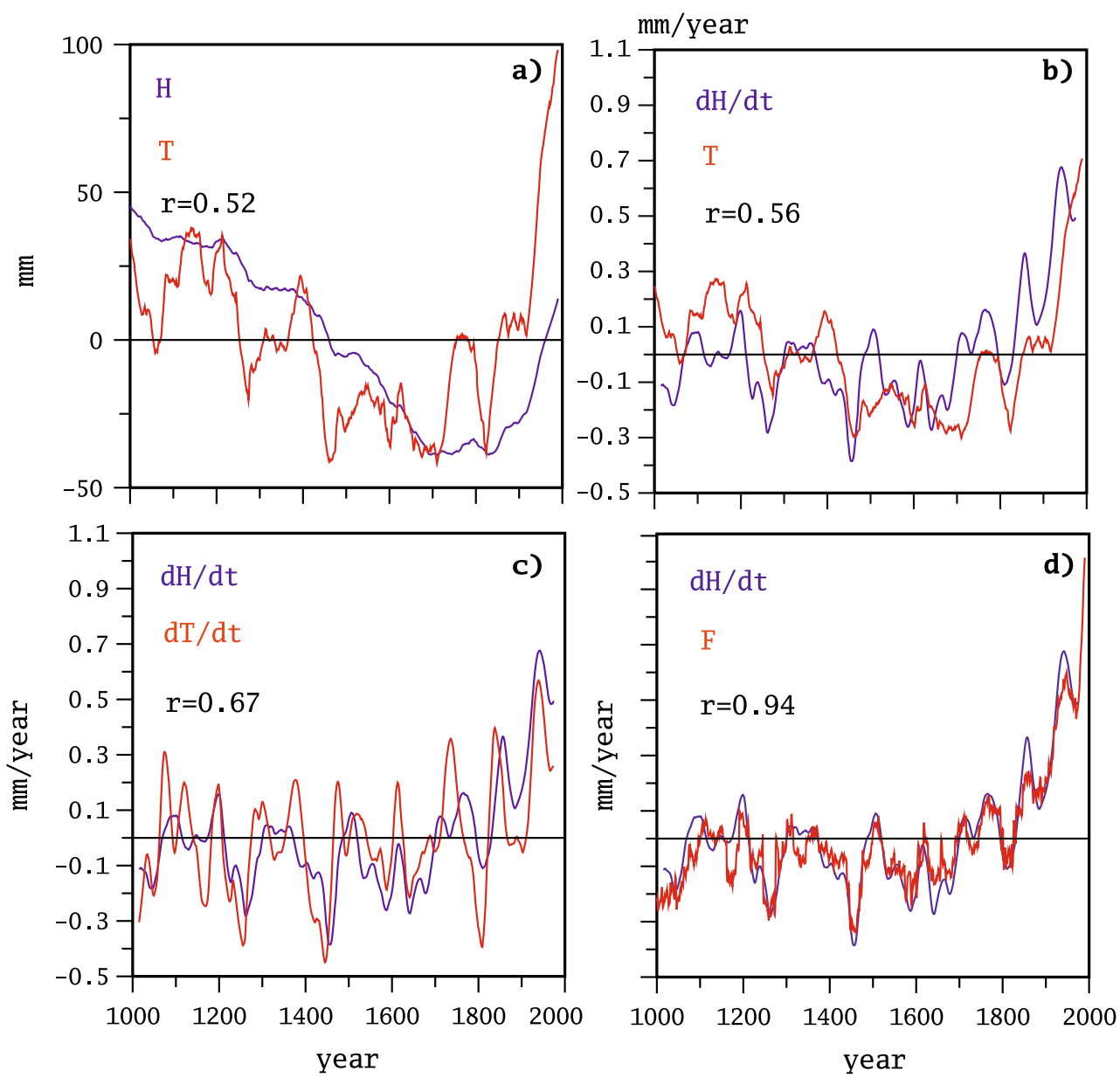


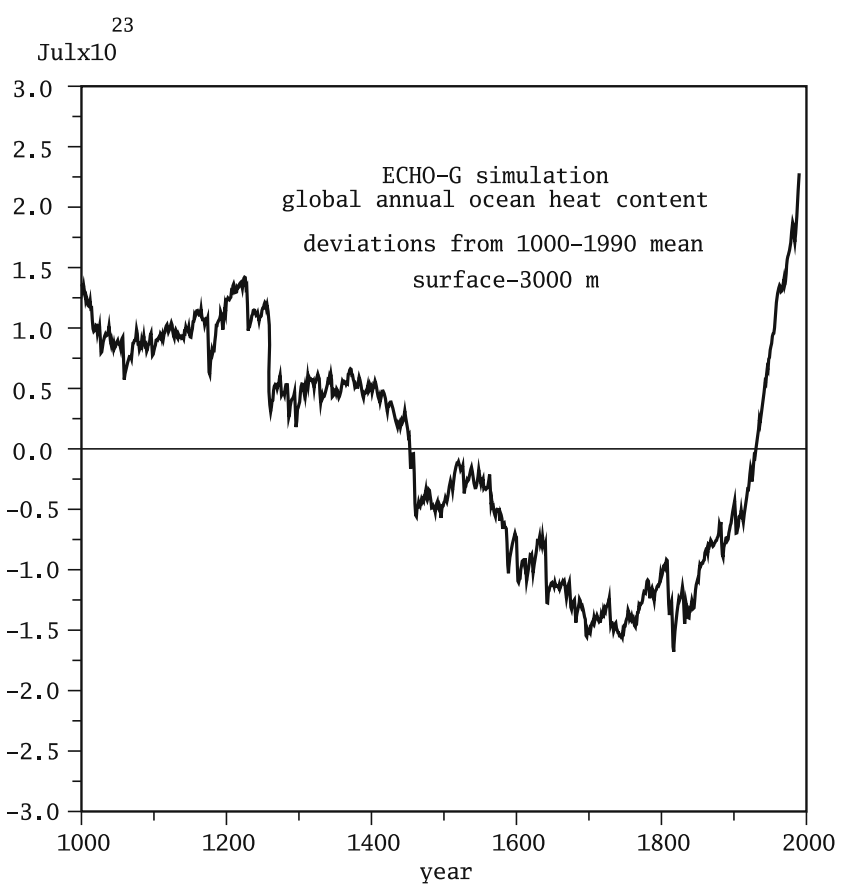

Fig. 2 Simulated evolution of the ocean heat content in the ECHO-G simulation in the past millennium between ocean surface and 3,000-m depth. This figure should be compared with that simulated with an energy balance model (Crowley et al. 2003; their Fig. 3). The sharp drops in heat content around 1260 and 1820 are caused by the large volcanic eruptions

Figure 1 additionally shows paired time series from the ECHO-G simulation that form the basis of the linear statistical models presented in the previous section. The skill of each model along the whole simulation is assessed by the correlation between the respective predictor and predictand timeseries, indicated also within each of the panels in Fig. 1.

As expected from physical reasoning, the best match is found between the vertical heat-flux and the rate of sea-level change ( $r=0.94$; model 2). Assuming for the moment that the equation of state of seawater is linear on temperature and independent of pressure and salinity, and that heat capacity is independent of temperature and salinity as well, so that the thermal expansion coefficient would be just constant for all water masses, sea-water-specific volume would be proportional to temperature and, therefore, the rate of sealevel change would also be strictly proportional to the total heat flux. This ideal behaviour would bring about a simultaneous correlation of unity between sea-level rise and total heat flux. Because, in reality, deviations from this ideal behaviour occur, and the thermal expansion of water brought about by the heat-flux depends also on temperature and salinity, the correlation between the rate of sea-level change and heat-flux is not perfect. Also, the slow penetration of the heat-flux into the ocean induces a time lag between both variables since the thermal expansion undergone by an ocean layer depends on its temperature, pressure and salinity and, therefore, on the depth of the layer.

Next in terms of skill is the statistical relationship between the rate of change of temperature and the rate of change of sea level $(r=0.67)$. This illustrates probably that the ocean-heat flux is more closely connected to the rate of change of near-surface air temperature than to the temperature deviations themselves. This can be also expected from physical reasoning.

The third statistical model in terms of skill is Eq. 1, which employs global mean air temperature as predictor and the rate of sea-level change as predictand $(r=0.56)$. Both time series agree on multi-centennial timescales, but they show discrepancies at centennial and multidecadal timescales. This can be problematic because, in practice, the statistical model Eq. 1 is calibrated with observational data in the observational period at these timescales. Thus, the apparent agreement in a short calibration period may not be stable in time. This will be investigated further in the following sections.

Lastly, the worst statistical model is Eq. 3, based on global mean temperature as predictor and global mean sea-level as predictand $(r=0.52)$. It is noteworthy that, as all series are highly autocorrelated, a test for significance for these correlations is not straightforward.

The 1-year-lag autocorrelation for all these series is close to 0.99 . Taking as null-hypothesis that the time series are the result of an autoregressive process of order 1 with a 1-lag autocorrelation of 0.99 , the $95 \%$ significance level estimated by Monte Carlo realisations is 0.46 . Sub-sampling the time series, for instance, 1 year per decade, does not reduce the degree of autocorrelation, which stays in the range 0.76 to 0.99 . A one-in30 sub-sampling reduces the autocorrelation of the time derivative of $T$ to almost zero, whereas the autocorrelation of the other time series remains higher than 0.6.

The practical applications of the statistical models that use $T$ or $\frac{d T}{d t}$ as predictors will be now analysed in more detail. The rationale behind this is that both predictors could be potentially better estimated in the real world from observations than the heat flux $F$. The process of estimating the regression parameter will be emulated in the virtual reality of the ECHO$G$ simulation, recalling that, in the climate model, only the thermosteric contribution is represented. As the simulation ends in year 1990, the pseudo-observational period for calibration is 1880-1990, instead of 1880 2000 as in the real observations. 

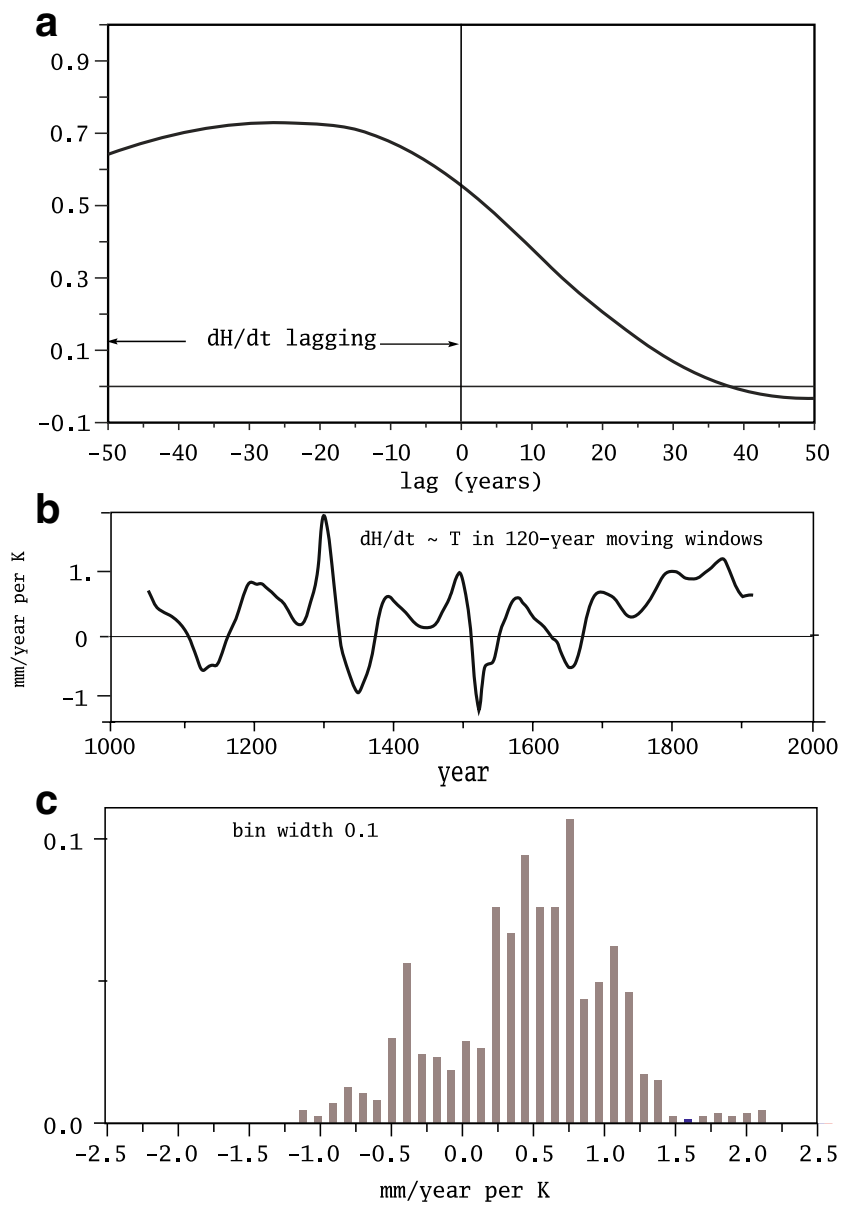

Fig. 3 a Lag-correlation function between global mean nearsurface air temperature and rate-of-change of global mean sea level in the millennium simulation with the model ECHO-G (model Eq. 1). b regression coefficient between global mean nearsurface air temperature and global mean sea-level rate of change (thermosteric component) in gliding windows of 120 years in the ECHO-G simulation of the past millennium. $\mathbf{c}$ histogram of the values depicted in $\mathbf{b}$

In the period 1880-1990, the least-mean-square error estimation of $a$ yields a value of $0.57 \mathrm{~mm} / \mathrm{year}$ per Kelvin, which amounts to about one third of the value estimated by Rahmstorf (2007a) in the simulation with the CLIMBER model, also considering only the thermosteric contribution. The correlation between $T$ and $\frac{d H}{d t}$ is, in this period, 0.68 . The estimation of the uncertainty bounds for this central value of $a$ is not straightforward, as both timeseries, predictor and predictand are strongly autocorrelated (lag-1 autocorrelation of 0.99 for both). The residuals of the regression also display a high degree of positive autocorrelation with a very low Durbin-Watson statistic of 0.002 . This indicates that the conditions to apply a standard leastsquare-error estimator of the regression coefficients are not fulfilled. These statistical caveats may explain the large differences between the estimation of $a$ in ECHO$\mathrm{G}$ and in CLIMBER, although model differences may also play a role. Holgate also found large differences in estimations of $a$ in the observational record when using either the first or the second half of the record (Holgate 2007). These are all indications that the relationship between $T$ and $\frac{d H}{d t}$ may be more complex than linear.

As mentioned in Section 1, it seems plausible that a potential relationship between global mean temperature and the rate of change of global mean sea-level could be lagged in time. This aspect can be also easily tested in the ECHO-G simulation by inspecting the lag-correlation function between these two variables (Fig. 3a). The correlation attains its maximum value when the global mean temperature is leading by about 25 years, which seems reasonable from a physical point of view. The observational timeseries would be too short to test whether this lag is realistic, although some hints of this can be discerned (see Fig. 3 in Rahmstorf 2007b), but it would be an interesting parameter to compare in other long climate simulations, once they become available.

The question arises as to whether the estimation of the regression parameter in model Eq. 1 is stable in time. The value of the regression parameter $a$ has been estimated in running segments of 120 -year lengths along the simulations. The resulting timeseries together with its histogram are displayed in Fig. $3 b$ and c. The possible values for the regression parameter cover a wide range, and they even show non-negligible periods with inverse relationships.

A similar analysis has been performed for the model using the rate of change of global temperature (model 4). There exists a consistent lag-relationship between the rate of temperature change leading the sea-level rate of change in the model by about 8 years. This lag changes little (by about 1 year) when estimated in both halves of the simulation, and therefore, it seems to be quite stable. The lag correlation function (Fig. 4) appears narrower than the corresponding lag correlation function between $d H / d t$ and $T$, and could be visually deduced from Fig. 1. The same time lag exists between the $d T / d t$ and the heat flux $F$, as the relationship between $F$ and the sea-level rate of change is, in the model, basically simultaneous. This means that the rate of change of temperature leads the heat flux into the ocean also by about 8 years.

The regression parameter attains in the last 120 years of the simulation a value of $35 \mathrm{~mm} / \mathrm{K}$ (e.g. mm/year per Kelvin/year), but it also varies when estimated in sliding 120-year windows along the simulation (Fig. 4b). However, although the range of variations is large, the regression parameter displays, most of the time, 


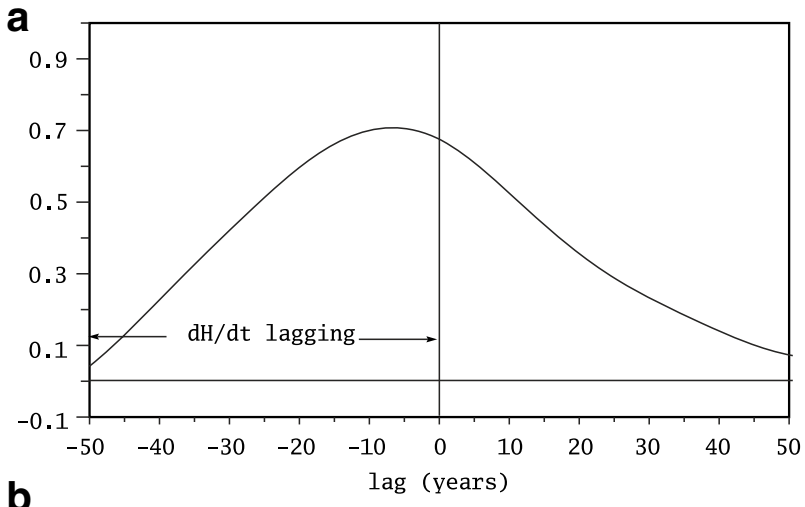

b

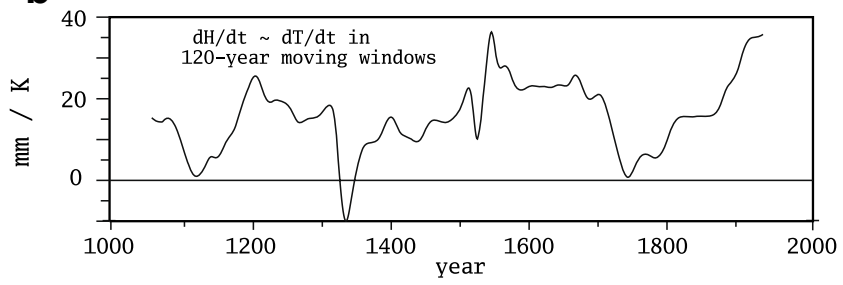

C

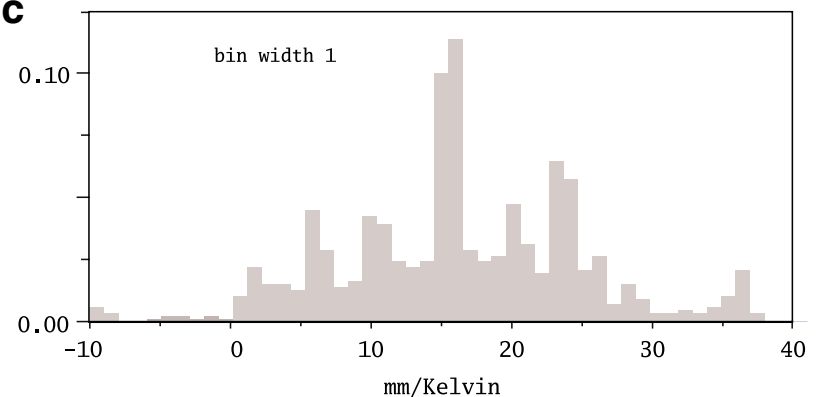

Fig. 4 a Lag-correlation function between the rate of change of the global mean near-surface air temperature and rate-of-change of global mean sea level in the millennium simulation with the model ECHO-G (model Eq. 4). b Regression coefficient between global mean near-surface air temperature and global mean sealevel rate of change (thermosteric component) in gliding windows of 120 years in the ECHO-G simulation of the past millennium. c Histogram of the values depicted in $\mathbf{b}$

positive values. The histogram derived from the timeseries displayed in Fig. 4b is shown in Fig. 4c. This histogram indicates that, also for this pair of variables, the uncertainty bounds for the regression estimated in a 120 -year period are substantial, even if at least the slope of their linear relationship is clearly positive.

In real applications, both predictors will have to be applied by fitting their respective linear models in a relatively short period of time - roughly 120 years and the resulting models will be used to predict the future behaviour of the rate of sea-level change in the following decades. This predictive skill can be also emulated in the context of the climate simulation. For this purpose, we consider a sliding window of 120-year

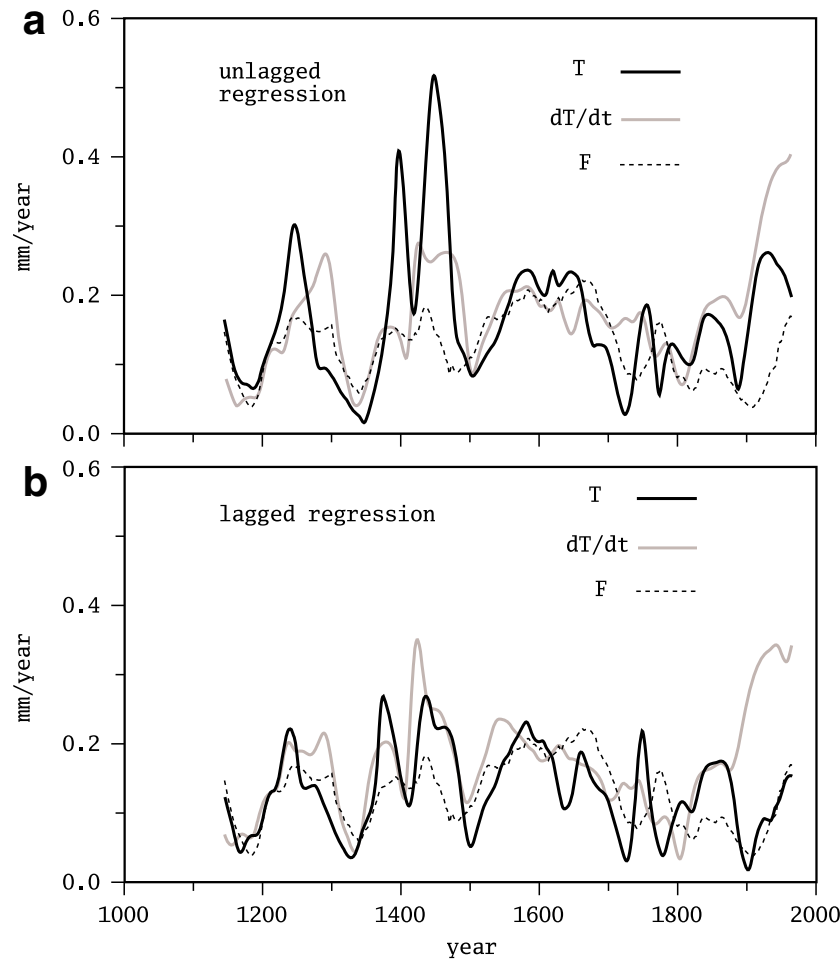

Fig. 5 a RMS in the estimation of the rate of sea-level change $\left(\frac{d H}{d t}\right)$ in sliding windows of 50 years along the climate simulation. Linear models using the global mean temperature $T$, its rate of change $\frac{d T}{d t}$ or the global mean heat-flux, calibrated in the 120year period previous to the 50-year sliding window, are used to estimate $\frac{d H}{d t}$, which is then compared to the value simulated by the climate model; $\mathbf{b}$ as in $\mathbf{a}$ but choosing the optimal lag for each predictor in the regression model

length where the linear models are calibrated, taking either $T$ or $\frac{d T}{d t}$ as predictors and $\frac{d H}{d t}$ as predictand. The predictand is estimated in the 50 years following the end of the sliding time window. The estimations can then be compared to the target variable simulated by the climate model, and a root-mean-squared (RMS) error for the length of the time window can be computed.

The results are displayed in Fig. 5a. For the sake of comparison, the RMS error obtained when using, in a similar manner, the heat-flux $F$ as predictor are also included. As expected, the heat-flux provides estimations with the lowest errors, with a time-mean value along the simulation of $0.12 \mathrm{~mm} / \mathrm{year}$. The rate of change of temperature $\frac{d T}{d t}$ yields a time-mean error of $0.16 \mathrm{~mm} / \mathrm{year}$, whereas the time-mean RMS incurred when using $\frac{d T}{d t}$ as predictor is $0.17 \mathrm{~mm} /$ year. The standard deviation of $\frac{d H}{d t}$ is $0.19 \mathrm{~mm} / \mathrm{year}$. The heat-flux is the best predictor almost always along the simulation, whereas $T$ and $\frac{d T}{d t}$ alternate as the worst predictor among these three. In 
general, $\frac{d T}{d t}$ performs slightly better than $T$, the differences not being very large.

The comparison of the RMS errors for the three predictors is, however, not completely fair, as the time lag between each predictor and these predictands is different. For instance, a regression model taking into account the 25-year lag to the sea-level rate of change may provide better results than the regression model assuming no lag between predictor and predictand. Similar to the results shown in Fig. 5a, Fig. 5b shows the mean-root-square errors along the simulation obtained when the regression models are set using the optimal lag relationship between predictor and predictand.

The differences between the unlagged and lagged regression are small for the rate of change of temperature (model Eq. 4) (now $0.17 \mathrm{~mm} /$ year), as the optimal lag was just 8 years. For the global mean temperature (model Eq. 1), the use of the optimal lag markedly reduces the prediction RMS to $0.13 \mathrm{~K} / \mathrm{mm}$. In the last sliding window at the end of the simulation, when the sea level is increasing at a rate of about $0.6 \mathrm{~mm} / \mathrm{year}$, the smallest error obtained among the three predictors is about $0.2 \mathrm{~mm} /$ year. It should be noted that, for the estimation of the lagged regression coefficient, the sliding window for the predictor was also shifted back in time with respect to the sliding window of the predictand, so that, also in this case, 120 years of data was available for the estimation-except for the beginning of the simulation. This would not be possible in the real world for two reasons: firstly, it would be much more difficult to identify the optimal lag in the short observational record, and secondly, the shorter overlapping period between the predictand and the lagged predictor will reduce the data available to calibrate the regression model even more.

\section{Conclusions}

Several simple linear models linking global mean sea level, global mean temperature, their rate of change and the heat-flux into the ocean have been tested in a long simulation of the past millennium with the climate model ECHO-G. These relationships could be potentially used to estimate empirically the future sealevel change based on the temperature changes simulated by climate models driven by different scenarios of emissions of greenhouse gases. This type of test performed in the virtual reality produced by climate models cannot prove whether a certain hypothesis, in this case the different statistical relationships, will hold in the real world as well. However, they can be used to falsify a particular hypothesis: if it is not fulfilled in a simple virtual reality, it will probably also fail in a more complex real world.

The sea level simulated by the model ECHO-G, for instance, only contains the contribution of the thermal expansion of the water column and disregards other contributions that are present in the real world as the melting of land ice. However, it seems plausible to assume that the existence of several and unrelated physical processes that link temperature and sea-level would hinder their description by a simple statistical linear model. In this sense, the conditions provided by the climate model to test a particular statistical relationship will be too optimistic.

Even in these conditions, it has been found that the best statistical model of the four explored here is the one that uses the ocean heat-flux as predictor. Unfortunately, the ocean heat-flux is a variable that is difficult to estimate in the real world, and of which long time series simply do not exist. Therefore, this close relationship is not useful to estimate empirically future sea-level changes.

The linear link between global mean temperature and the rate of change of global mean sea level (model Eq. 1) has turned out to be not reliable over the full time period in the context of this climate simulation; instead, for some periods, even inverse relationships were found to describe the simulated data best. The second predictor "rate of change of temperature", used in model Eq. 4, analysed here in more detail, did not show markedly better results. For both predictors, there exist periods in the simulation where the prediction errors are very large. A statistical analysis using the rather short, and heavily smoothed, observational record, which suffers from the strong temporal correlation and strong linear trends, it seems compulsory to submit statistical model to a careful test with simulated data, ideally stemming from long climate simulations performed with several different models.

Acknowledgement F. G-R was partially funded by the Spanish Ministerio de Educación y Ciencia through grant REN-200204584-CLI.

Open Access This article is distributed under the terms of the Creative Commons Attribution Noncommercial License which permits any noncommercial use, distribution, and reproduction in any medium, provided the original author(s) and source are credited. 


\section{References}

Allen MR, Smith LA (1996) Monte Carlo SSA: detecting irregular oscillations in the presence of coloured noise. J Climate 9:3373-3404

Antonov JI, Levitus S, Boyer TP (2005) Thermosteric sealevel rise. Geophys Res Lett 32:L21602. doi:10.1029/ 2005GL023112

Cazenave A, Nerem RS (2004) Present-day sea-level change: observations and causes. Rev Geophys 42. doi:10.1029/ 2003RG00139

Crowley TJ (2000) Causes of climate change over the past 1000 years. Science 289:270-277

Crowley TJ, Baum SK, Kim K-Y, Hegerl GC (2003) Modeling ocean heat content changes during the last millennium. Geophys Res Lett 30. doi:10.1029/2003GL017801

Etheridge DM, Steele LP, Langenfelds RL, Francey RJ, Barnola J-M, Morgan VI (1996) Natural and anthropogenic changes in atmospheric $\mathrm{CO}_{2}$ over the last 1000 years from air in Antarctic ice and firn. J Geophys Res 101(D2): 4115-4128

Etheridge DM, Steele LP, Francey RJ, Langenfelds RL (1998) Atmospheric methane between 1000 A.D. and present: evidence of anthropogenic emissions and climatic variability. J Geophys Res 103(D13):15979-15994

Fofonoff NP, Millard RC Jr (1883) Algorithms for computation of fundamental properties of seawater. UNESCO technical papers in marine science no. 44. UNESCO, Paris, p 53

González-Rouco JF, Beltrami H, Zorita E, von Storch H (2006) Simulation and inversion of borehole temperature profiles in surrogate climates: spatial distributions and surface coupling. Geophys Res Lett 33:L01703. doi:10.1029/ 2005GL024693

Gregory JM, Church JA, Boer GJ, Dixon KW, Flato GM, Jackett DR, Lowe JA, O'Farrell SP, Roeckner E, Russell GL, Stouffer RJ, Winton M (2001) Comparison of results from several AOGCMs for global and regional sea-level change 1900-2100. Clim Dyn 18:225-240

Holgate S (2007) Comment on 'a semi-empirical approach to projecting future sea-level rise'. Science. doi:10.1126/ science 1143286

Jevrejeva S, Grinsted A, Moore JC, Holgate S (2006) Nonlinear trends and multiyear cycles in sea level records. J Geophys Res 111:C09012. doi:10.1029/2005JC003229

Kaufmann RK, Stern DI (2002) Cointegration analysis of hemispheric temperature relations. J Geophys Res 107. doi:10.1029/2000JD000174

Katsman CA, Hazeleger W, Drijfhout SS, van Oldenborgh GJ, Burgers G (2008) Climate scenarios of sea level rise for the northeast Atlantic Ocean: a study including the effects of ocean dynamics and gravity changes induced by ice melt. Clim Change. doi:10.1007/s10584-008-9442-9

Legutke S, Voss R (1999) The Hamburg atmosphere-ocean coupled circulation model ECHO-G. Deutches Klimarechenzentrum Technical Report 18. DKRZ, Hamburg

Lee TCK, Zwiers FW, Tsao M (2008) Evaluation of proxy-based millennial reconstruction methods. Clim Dyn 31:263-281. doi:10.1007/s00382-0007-0351-9

Meehl GA, Stocker T, Collins W, Friedlingstein P, Gaye A, Gregory JM, Kitoh A, Knutti R, Murphy J, Noda A, Raper S, Watterson IG, Weaver AJ, Zhao Z-C (2007) Global climate projections. In: Solomon S, Qin D, Manning M, Chen Z, Marquis M, Averyt KB, Tignor M, Miller HL (eds) Climate change 2007: the physical science basis. Contribution of working group I to the fourth assessment report of the intergovernmental panel on climate change. Cambridge University Press, Cambridge

Montoya M, Griesel A, Levermann A, Mignot J, Hofmann M, Ganopolsky A, Rahmstorf S (2005) The earth system model of intermediate complexity CLIMBER-3 $\alpha$. Part I: description and performance for present-day conditions. Clim Dyn 25:237-263

Osborn TJ, Raper SCB, Briffa KR (2006) Simulated climate change during the last 1,000 years: comparing the ECHO-G general circulation model with the MAGICC simple climate model. Clim Dyn 27:185-197

Russell GL, Miller JR, Rind D, Ruedy RA, Schmidt GA, Sheth S (2000) Comparison of model and observed regional temperature changes during the past 40 years. J Geophys Res 105:14891-14898

Schmith T, Johansen S, Thejll P (2007) Comment on 'a semiempirical approach to projecting future sea-level rise'. Science. doi:10.1126/science1143286

Rahmstorf S (2007a) A semi-empirical approach to projecting future sea-level rise. Science 315:368-370

Rahmstorf S (2007b) Response to comment on 'a semiempirical approach to projecting future sea-level rise'. Science. doi:10.1126/science1143286

von Storch H, Zorita E, Jones J, Dimitriev Y, González-Rouco F, Tett SFB (2006) Reconstructing past climates with noisy data. Science 306:679-682

von Storch H, Zorita E, González-Rouco JF (2008) Assessment of three temperature reconstruction methods in the virtual reality of a climate simulation. Int J Earth Sci (Geol Rundsch). doi:10.1007/s00531-008-0349-5

Zorita E, González-Rouco F, von Storch H (2007) Comments on 'testing the fidelity of methods used in proxy-based reconstructions of past climate'. J Climate 20:3693-3698 